\title{
Design a 90 Degree Splitter WDM using Plasmonic Technique
}

\author{
Mohammed Nadhim Abbas \\ Electrical Engineering Department, \\ College of Engineering, University of Baghdad, \\ Baghdad, Iraq
}

\author{
Ahmed Abdulredha Ali \\ Electrical Engineering Department, \\ College of Engineering, University of baghdad
} Baghdad, Iraq

\begin{abstract}
The two channels of plasmonic filter structure are selective based on a nanocavity that proposed and numerically simulated by using the finite element method by using COMSOL4.4 software package The required filtered wavelength can be investigated by selecting an appropriate length of the nanocavity and refractive index of dielectric that filled nanocavity. Two output channels structure based on two perpendicular nanocavities that proposed to design a subwavelength plasmonic splitter, and demultiplexer operating around $770 \mathrm{~nm}$ and $900 \mathrm{~nm}$ with the maximum transmittance of the two bands is $79 \%$ for channell and $84 \%$ for channel2.Three materials used to build structure ,metal used as a silver and two types of dielectric quartz with refractive index 1.5 and erbium with refractive index 1.45 .
\end{abstract}

\section{General Terms}

WDM , plasmonic (WDM)

\section{Keywords}

Plasmonics, Surface plasmon polration, Localized surface plasmon, resonance wavelength,

\section{INTRODUCTION}

Electromagnetic waves incident on metal-dielectric interfaces and combined to propagate free electron oscillations in the metals, known as surface plasmon polaritons (SPPs), are considered as the technique that help to realization of highly integrated optical circuits because they can considerably the solution for the classical diffraction limit of light and manipulate light on the nano scale [1-4]. Metaldielectric-metal (MDM) waveguide have special features because of its high confinement of the light, simplicity, and convenience for fabrication and integration into optical circuits. The form of functional plasmonic MDM structures have been implemented and fabricated, such as U-shaped waveguides [5], splitters [6], switches [7], [8], Y-shaped combiners [9], couplers [10], and M-Z interferometers [11], [12]. There is recognition that the communication between electronic systems constitute the main constraint to increase system speed and speed of data transfer. There is optimism that the optical communication hold great promise in eliminating congestion in the current connections because of characteristics that stem from the inherent parallelism of optics. Wavelength division technology [13]. Wavelength selection technique is one of the main technologies in the fields of optical communication. Candidates on the basis of nanocavities, [14], coupled cavity side [15], teeth or heel structure [16] - [17] has recently been investigated. However, All filters wavelength in previous research, a structure in the form of a cavity or stub which often reflect either the passband or stop it. The reflected or transmitted waves in such structures are in the same a single channel is difficult for you to be separated from each other. However, the WDM, which is divide the light into several channels, and is a unit of light divider is the basic unit in WDM system. Recently, Gan et al. [18], [19] Suggested a plasmonic surface-wave divider based on a bidirectional subwavelength slit, and suggested split spectrally selective with metal insulating metal surface plasmon waveguide. However, all the dividers structures mentioned above have high volumes over several waves. Given the perspective of the integration of the components of the traitor within several micrometers, and people need to reduce the size of devices to meet the demand of high integration.. In this paper, subwavelength SPP splitters and demultiplexers structures based on MIM nanocavities are proposed and demonstrated numerically by using the finite element method (FEM) in software package COMSOL4.4. The good feature of plasmonic demultiplexer used to solve huge size of optical integrated circuit problem and large size of optical (WDM) the principle of plasmonic should be used . With the development of artificial fabrication, plasmonic (WDM) devices may be fabricated and applied in future alloptical communications and integrated optical circuits. As an important plasmonic waveguide, the metal-dielectric-metal (MIM) structure has high confinement of light with an acceptable length for SPP propagation. Plasmonic wavelength demultiplexers (WDMs), which can filter specific wavelengths in different channels, will play very important role in the future all-optical communication systems. Figure 1 shows the two outputs plasmonic (WDM)

\section{NANOCAVITY MODEL}

Figure (1) shows the structure schematic of the nanocavity composed of three wide waveguides and two a narrow waveguide filled with quartz and erbium material. The structure can be deals with three MIM waveguides with different widths. Because the narrow width of MIM waveguide is much smaller than that of the wide MIM waveguide; here, has been called the narrower waveguide as nanocavity.

The dielectric constant $\mathcal{E}_{m}$ of silver can be compute based on applicable experiment data and a more detailed fit of the data reported by Johnson and Christy for silver [22]. The absorption band noted in the imaginary part of the dielectric constant between $0.3 \mu \mathrm{m}$ and $0.4 \mu \mathrm{m}$ that's because of absorption which is occurred in d-band electrons. The effective index of the waveguide decreases with the increasing of the width of waveguide $w$ at the same wavelength. Therefore, the effective index neff 2 of the nanocavity part can be larger than neffl of the wide MIM waveguide part due to the difference of the width of the two waveguides and mediums filled in. The waves will propagated into the nanocavity because its higher effective index, when SPP waves propagate along the interface between metal and air. 
The wave transmitted into the nanocavity will be partly reflected at two ends of the nanocavity, because of the index differences between neff 2 and neffl.

\section{EFFECT LENGTH OF NANOCAVITY}

It can be seen that the peak wavelength is proportional to the length of the nanocavity, which can be seen in the simulation results (see figures $2 \mathrm{a}, \mathrm{b}$ ) in section five (using COMSOL4.4 software). Based on the theoretical analysis fore effective refractive index, the operating wavelength of the proposed structure can be effectively modulated by altering the length of the nanocavity, and the effective index of surface plasmon polaritons (SPPs) in the cavity, which is determined by its width. Has been noted the transmission wavelength of each channel has been a linear relationship with length of the nanocavity and exhibits redshift while increasing the value of $L$, which is in conformity with the solution of Eq. below [21].

$\lambda_{m}=\frac{2 n_{e f f} L}{(m-\phi r / \pi)}$

Therefore, the required wavelength in each channel is obtained by properly selecting the specific parameters of the structure, such as the length or width of the nanocavity.

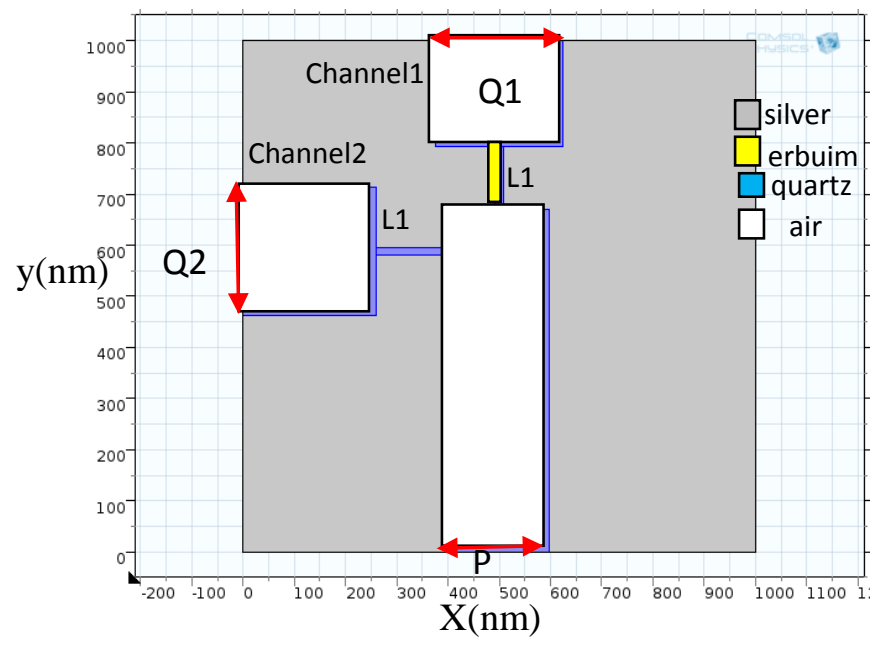

Fig. (1): schematic Wavelength $(\mu \mathrm{m}) \quad$ plasmonic splitter and demultiplexer sucucure (cop view).

\section{EFFECT WIDTH of NANOCAVITY}

Above equation indicates the resonance wavelength behavior of each nanocavity (channel of the demultiplexing structure) depends mainly on two parameters: the length of the nanocavity, and the effective index of SPPs in the nanocavity, which is determined by its width. To fully understand how the width of the nanocavity structure influences on the SPPs propagation, Has been noted the width $w$ at various wavelengths of the incident light are calculated and shown in figures $3 \mathrm{a}, \mathrm{b}$.

One can see that the resonance wavelength of surface plasmon decreases with increasing width $w$ of nanocavity. The width of nanocavity has been noted very narrow, but the light propagate through it ,because the input light should be ppolarize or TM polarize, therefore create surface plasmon polariton and propagated through the nanocavity despite the wavelength of electromagnetic wave greater than the narrow nanocavity. In the classical optical communications the incident light can not propagate through nano scale because diffraction limit. The problem of diffraction limit can overcome by plasmonic techniqe that used in this paper.

\section{RESULTS AND DISCUSSIONS}

The electric field $E$ and magnetic field $H$ are calculated along the interface of (WDM) silver metal with dielectric in the form MDM waveguide. It can be seen that the intensity of electric field $E$ is maximum value at the resonance wavelength and decreased exponentially to the minimum value away from interface. In this section will show the propagations of field $E$ and the demultipexing effect for two monochromatic waves with different wavelengths of $770 \mathrm{~nm}$ and $900 \mathrm{~nm}$ launched into the $1 \times 2$ wavelength demultiplexing structure. However, one can see that the wave with the wavelength of $770 \mathrm{~nm}$ is just passing through the nanocavity cavity of channel 1 , while the wave with the wavelength of $900 \mathrm{~nm}$ is just transmitting from the nanocavity of channel 2. Figure $(4 a, b)$ depict the field distributions of normal electric field $\mathrm{E}$ with launching continuous waves at $770 \mathrm{~nm}$ and $900 \mathrm{~nm}$, for channel one and two respectively. Figure (4) as an application of the wavelength-selecting properties, this aperture can be exploited to construct a nano plasmonic wavelength demultiplexer. The field distributions are in good agreement with the transmission spectra in figure (5) .

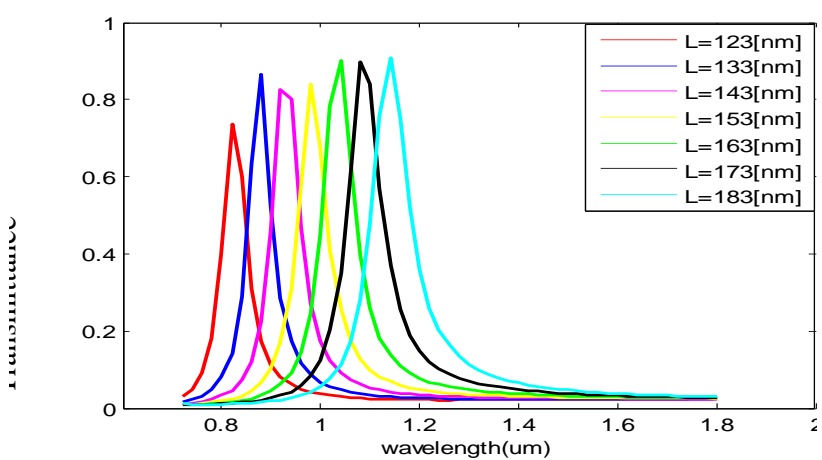

Fig . (2,a): Centeral wavelength at deferent length of nanocavity

The following table represent the length of nanocavity versus resonance wavelength

Table 1.length of nanocavity versus resonance wavelength

\begin{tabular}{|c|c|}
\hline Length of nanocavity (nm) & Resonance wavelength \\
\hline 123 & 0.82 \\
\hline 133 & 0.88 \\
\hline 143 & 0.92 \\
\hline 153 & 0.98 \\
\hline 163 & 1.04 \\
\hline 173 & 1.08 \\
\hline 183 & 1.14 \\
\hline 193 & 1.2 \\
\hline 203 & 1.24 \\
\hline 213 & 1.3 \\
\hline
\end{tabular}




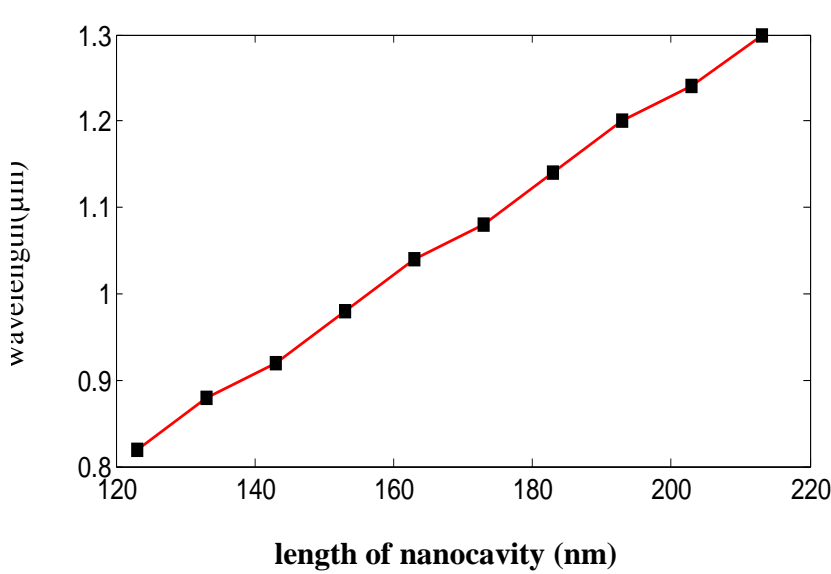

Fig . (2,b): wavelength function of length of nanocavity

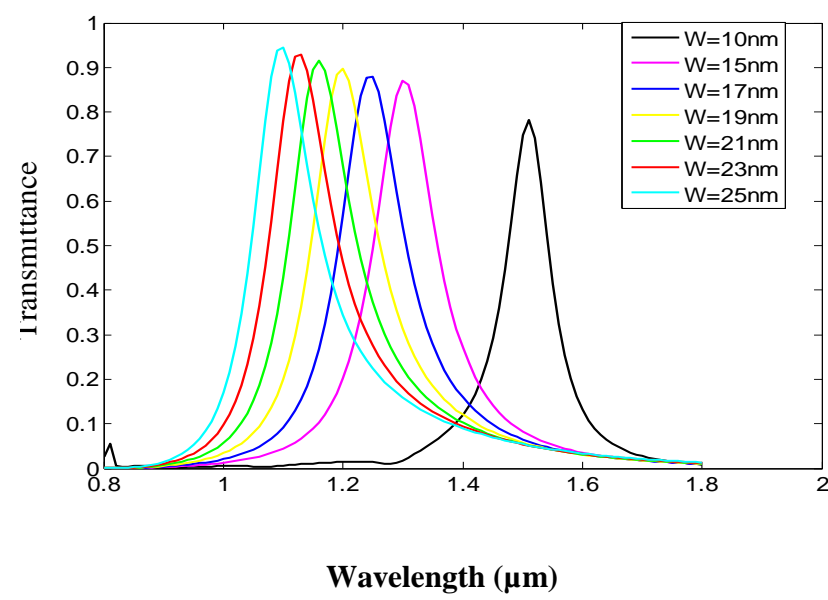

Fig - (3,a) Relationship the wavelength of the incident light and width of nanocavity

The following table data for the width of nanocavity versus resonance wavelength.

Table 2. Width of nanocavity versus resonance wavelength

\begin{tabular}{|c|c|}
\hline Width of nanocavity $(\mathbf{n m})$ & Resonance wavelength \\
\hline 10 & 1.51 \\
\hline 15 & 1.29 \\
\hline 17 & 1.25 \\
\hline 19 & 1.2 \\
\hline 21 & 1.16 \\
\hline 23 & 1.13 \\
\hline 25 & 1.1 \\
\hline 30 & 1.04 \\
\hline
\end{tabular}

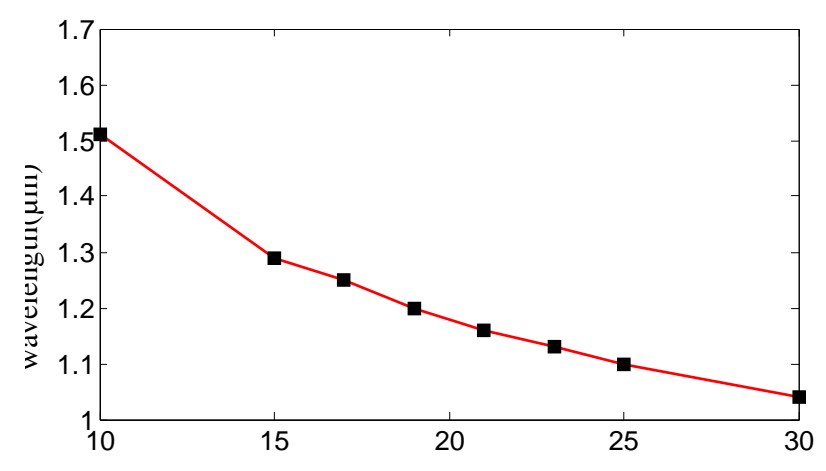

Width of nanocavity (nm)

Fig . (3,b): Inversely relationship between width of nanocavity and wavelengths

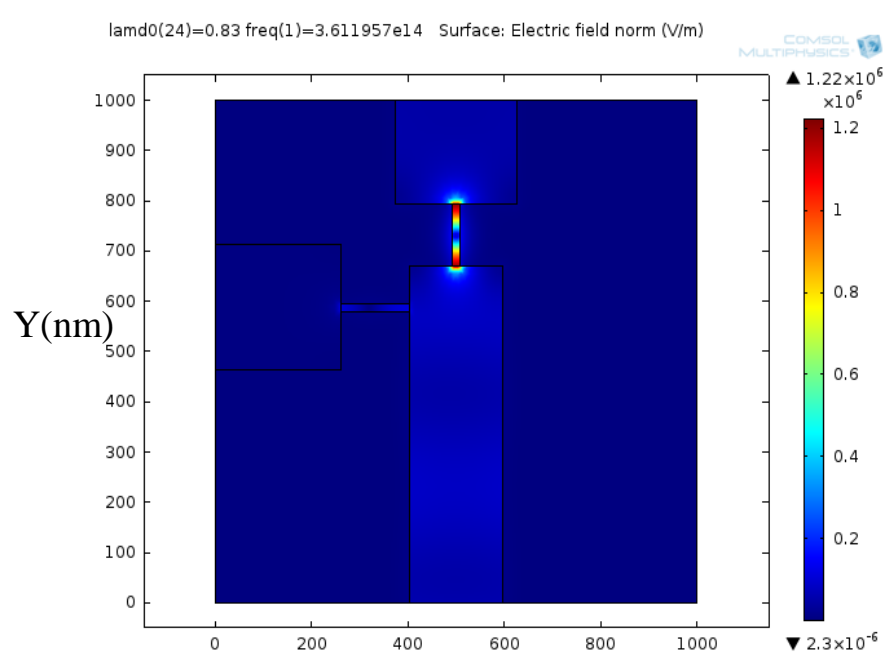

Fig . (4a)The contour profiles of surface electric field norm $E$ of the wavelength demultiplexing structure at $770 \mathrm{~nm}$

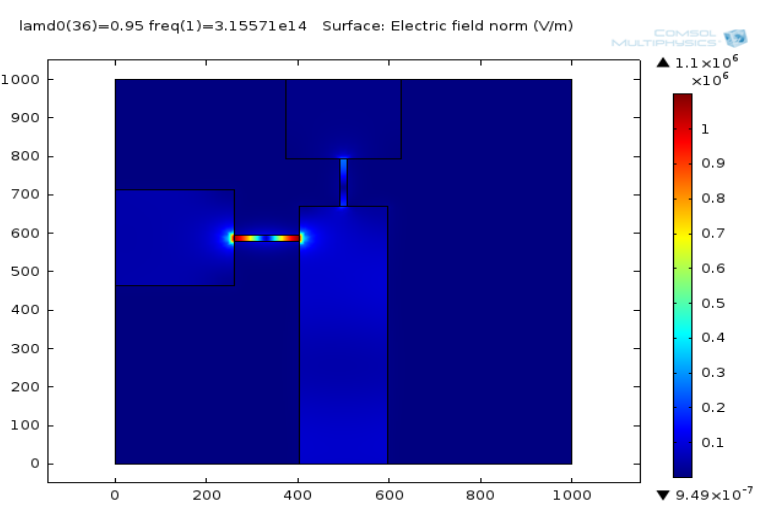

$$
\mathrm{X}(\mathrm{nm})
$$

Fig . (4b)The contour profiles of surface electric field norm $E$ of the wavelength demultiplexing structure at 900 nm 


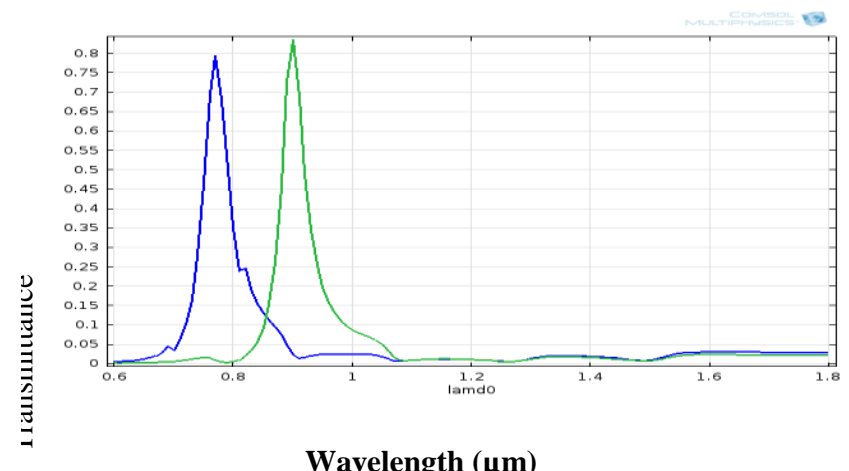

Wavelength $(\mu \mathrm{m})$

Fig - (5): Transmission spectra of the two output channels plasmonic splitter and demultiplexer structure .

\section{CONCLUSION}

In this paper, the resonance electric field of the light that propagated in the nanocavity of the plasmonic demultiplexer is investigated, when changing the nanocavity length and width. From simulation results, it is found that the charge density is maximum at the resonance wavelength cause the electric field to be enhanced and decayed exponentially . . Has been conclude the two output channels selective plasmonic filter structure dependent on the distance and angle between them. The required filtered wavelength can be conclude dependent on an appropriate selecting the length of nanocavity. Two output channels' structure is proposed to design a subwavelength plasmonic splitter and demultiplexer, based on two nanocavity waveguides operating around 770 $\mathrm{nm}$ and $900 \mathrm{~nm}$ at special dimensions for resonators and ,there is possibility to chan Wavelength $(\mu \mathrm{m})$ and width of resonators and then Wavelength $(\mu \mathrm{m})$ FEM simulation results reveal that this new structure is suitable for wideband wavelengths demultiplexing. The transmission spectra at the outputs of the two channels. One can see that channel1 and channel 2 are selected $770 \mathrm{~nm}$ and $900 \mathrm{~nm}$ bands with the length nanocavity of channel1 is $L 1 \mathrm{~nm}$ and channel2 is $L 2 \mathrm{~nm}$ as shown in figure (1). The transmission bands of $770 \mathrm{~nm}$ and $900 \mathrm{~nm}$ are selected, and the maximum transmittance of the two bands is $79 \%$ for channel1 and $84 \%$ for channel2. From the work conclude the small dimensions of proposed structures best choice for the fabrication of all-optical integrated devices and circuits for optical computing and communication, especially in WDM systems in the nanoscale .

\section{REFERENCES}

[1] W. L. Barnes, A. Dereux, and T. W. Ebbesen, "Surface plasmon subwavelength optics," Nature 424(6950), 824830 (2003).

[2] S. I. Bozhevolnyi, V. S. Volkov, E. Devaux, J. Y. Laluet, and T. W. Ebbesen, "Channel plasmon subwavelength waveguide components including interferometers and ring resonators," Nature 440(7083), 508-511 (2006).

[3] D. Gramotnev and S. Bozhevolnyi, "Plasmonics beyond the diffraction limit," Nat. Photonics 4(2), 83-91 (2010).

[4] Hua Lu, Xueming Liu,* Yongkang Gong, Dong Mao, and Leiran Wang "Enhancement of transmission efficiency of nanoplasmonic wavelength demultiplexer based on channel drop filters and reflection nanocavities" July 2011 / Vol. 19, No. 14 / OPTICS EXPRESS 12885

[5] T. Lee and S. Gray, "Subwavelength light bending by metal slit structures,”Opt. Exp., vol. 13, pp. 9652-9659, 2005.

[6] G. Veronis and S. Fan, "Bends and splitters in metaldielectric-metal subwavelength plasmonic waveguides," Appl. Phys. Lett, vol. 87, p. 131102, 2005.

[7] Z. Yu, G. Veronis, S. Fan, and M. L. Brongersma, "Gain-induced switching in metal- dielectric-metal plasmonic waveguides," Appl. Phys. Lett, vol. 92, p. 041117, 2008.

[8] C. J. Min and G. Veronis, "Absorption switches in metaldielectricmetal plasmonic waveguides," Opt. Exp., vol. 17, pp. 10757-107๔a nnnก

[9] H. Gao, H. Shi, C Wavelength $(\mu \mathrm{m}) \quad$ I. Deng, Y. Lv, X. Lin, and H. Yao, "Surface plasmon polariton propagation and combination in Y-shaped metallic channels," Opt. Exp., vol. 13, pp. 10795-10800, 2005.

[10] H. Zhao, X. Huang, and J. Huang, "Novel optical directional coupler based on surface plasmon polaritons," Physica. E, vol. 40, pp. 3025-3029, 2008.

[11] B. Wang and G. Wang, "Surface plasmon polariton propagation in nanoscale metal gap waveguides," Opt. Lett, vol. 29, pp. 1992-1994, 2004.

[12] Z. Han, L. Liu, and E. Forsberg, "Ultra-compact directional couplers and Mach-Zehnder interferometers employing surface plasmon polaritons," Opt. Commun, vol. 259, pp. 690-695, 2006.

[13] Feng Zhao1, Yun Zhang, Jizuo Zou, Wei Jiang, Zhong Shi, Xuegong Deng, Jie Qiao, Xuegong Deng, Gary C. Marsden, Bipin Bihari, and Ray Chen "Wavelength division multiplexers/demultiplexers for highthroughput optical links" Radiant Photonics Inc., Kramer Ln, Austin TX 78758, USA

[14] W. Lin and G. Wang, "Metal heterowave guide superlattices for a plasmonic analog to electronic bloch oscillations," Appl. Phys. Lett, vol. 91, p. 143121, 2007.

[15] Q. Zhang, X.-G Huang, X.-S. Lin, J. Tao, and X.-P Jin, "A subwavelength coupler-type MIM optical filter," Opt. Exp., vol. 17, pp. 7549-7554, 2009.

[16] X.-S. Lin and X.-G. Huang, "Tooth-shaped plasmonic waveguide lters with nanometeric sizes," Opt. Lett, vol 33, pp. 2874-2876, 2008.

[17] Y. Matsuzaki, T. Okamoto, M. Haraguchi, M. Fukui, and A. Nakagaki, "Characteristics of gap plasmon waveguide with stub structures," Opt. Exp., vol. 16, pp. 16314 $16325,2009$.

[18] Q. Gan, B. Guo, G. Song, L. Chen, Z. Fu, Y. J. Ding, and F. J. Bartoli, "Plasmonic surface-wave splitter," Appl. Phy. Lett., vol. 90, p. 161130, 2007.

[19] Q. Gan, Z. Fu, Y. J. Ding, and F. J. Bartoli, "Bidirectional subwavelength slit splitter for $\mathrm{THz}$ surface plasmons," Opt. Exp., vol. 15, p. 18050, 2007.

[20] P.B.Johnson, R.W.Christy, Phys. Rev. B 6, 4370 (1972)

[21] Feifei Hu and Zhiping Zhou," Wavelength filtering and demultiplexing structure based on aperture-coupled plasmonic slot cavities" J. Opt. Soc. Am. B / Vol. 28, No. 10 / October 2011

[22] P.B.Johnson, R.W.Christy, Phys. Rev. B 6, 4370 (1972). 